\title{
Screening and identification of Lynch syndrome: a systematic review of the frequency of Lynch syndrome-associated clinicopathologic and molecular characteristics in Lynch syndrome gynecologic cancers
}

\author{
Zihui Yang ${ }^{1,2}$, Xinyu Liu $^{3}$, Xi Yang ${ }^{1,2}$, Qin-Ping Liao ${ }^{1,2} \wedge$ \\ ${ }^{1}$ School of Clinical Medicine, Tsinghua University, Beijing, China; ${ }^{2}$ Department of Obstetrics and Gynecology, Beijing Tsinghua Changgung \\ Hospital, School of Clinical Medicine, Tsinghua University, Beijing, China; ${ }^{3}$ Department of Reproductive Medicine, Shenyang 204 Hospital, \\ Shenyang, China \\ Contributions: (I) Conception and design: QP Liao, Z Yang; (II) Administrative support: QP Liao; (III) Provision of study materials or patients: X Liu, \\ X Yang; (IV) Collection and assembly of data: Z Yang, X Liu; (V) Data analysis and interpretation: X Yang, QP Liao; (VI) Manuscript writing: All \\ authors; (VII) Final approval of manuscript: All authors. \\ Correspondence to: Qin-Ping Liao. School of Clinical Medicine, Tsinghua University, Beijing 100084, China; Department of Obstetrics and Gynecology, \\ Beijing Tsinghua Changgung Hospital, School of Clinical Medicine, Tsinghua University, Beijing 102218, China. Email: qinping_liao@163.com.
}

Background: This study aimed to investigate the frequency of Lynch syndrome-associated clinicopathologic and molecular characteristics in Lynch syndrome gynecologic cancers.

Methods: A systematic literature search was conducted in the literature databases (Medline, CINAHL, EMBASE, Google Scholar, Cochrane Library, and Clinicaltrials.gov) to identify the studies describing clinicopathologic characteristics, MMR protein immunohistochemistry and/or MSI, MLH1 methylation, and genetic testing in Lynch syndrome gynecologic cancer patients.

Results: A total of 24 of the evaluated studies that met the inclusion criteria were identified. A clinicopathological examination confirmed 242 endometrial cancer, 17 clear cells endometrial cancer, 35 serous endometrial cancer, 30 mixed and 21 other endometrial cancer. Thus, a total of 345 endometrial cancer was confirmed from the screening of 1,317 gynaecological cancer. However, the morphological analysis demonstrated 236 patients with endometrial cancer associated with Lynch syndrome. The frequency of confirmed LS with endometrial cancer was $68.40 \%$. At diagnosis, the median age was $49.94 \pm 4.34$ years, and the average BMI was $26.07 \pm 3.77 \mathrm{~kg} / \mathrm{m}^{2}$. Endometrioid histology and stage I disease were the most frequent at $70.97 \%$ and $71.19 \%$ histological type and FIGO stage, respectively. Similarly, morphological and histological analysis demonstrated a higher degree of grade I cancer (47.28) and lymphovascular invasion (56.52\%), respectively.

Discussion: Lynch syndrome-associated clinicopathologic and molecular characteristics occur significantly in Lynch syndrome gynecologic cancers and may improve risk stratification and triaging of gynecologic cancers for genetic testing.

Keywords: DNA mismatch repair protein; gynecologic cancer; immunohistochemistry; Lynch syndrome (LS); MLH1 methylation

Submitted May 21, 2021. Accepted for publication Sep 03, 2021.

doi: $10.21037 /$ tcr-21-677

View this article at: https://dx.doi.org/10.21037/tcr-21-677

^ ORCID: 0000-0002-4412-8474. 


\section{Introduction}

Lynch syndrome (LS) is an autosomal dominant hereditary syndrome that increases the risk of the early development of multiple cancers, including colorectal (CRC), gynecologic, urothelial, and biliary tract cancers (1). The syndrome is implicated in $5 \%$ of endometrial cancers (EC), with a lifetime risk of $40-60 \%(2,3)$.

LS has also been linked to cancers of the endocervix (4). The syndrome results from inactivating germline mutations in genes that encode mismatch repair (MMR) proteins. Pathogenic MMR gene mutations associated with LS typically involve four primary genes, including MLH1, PMS2, MSH2, and MSH6 (1,3). MSH2 expression can also be lost due to epigenetic silencing resulting from the germline deletion of the EPCAM gene $(5,6)$.

Immunohistochemistry (IHC) of proteins encoded by the DNA MMR genes and microsatellite instability (MSI) are becoming the mainstay of LS molecular screening, supplementing the family history-based clinical criteria such as Amsterdam II and Bethesda (7-9). IHC tests the expression of MMR proteins in tumour tissue. MLH1 and PMS2 are heterodimers. MLH1 mutation, therefore, results in the loss of expression of both MLH1 and PMS2. Similarly, MSH2 and MSH6 are heterodimers, with MSH2 transformation resulting in the loss of expression of MSH6 $(3,7,10)$. However, isolated mutations of PMS2 and MSH6 can result in the isolated loss of expression of these genes. MLH1 failure can result from epigenetic silencing through promoter hypermethylation (10). Detection of MLH1 methylation with intact MSH2/MSH6 expression suggests a sporadic origin of a tumour $(10,11)$.

MSI testing is a polymerase chain reaction-based (PCR) test that assesses DNA for the presence of somatic changes. The presence of MSI in at least two loci is pathogenic (MSIHigh) (10). However, MSI has low sensitivity in MSH2 and MSH6 mutations, with a sensitivity of $55 \%$ and $77 \%$ in MSH2 and MSH6, versus 90\% in MLH1/PMS2 (12). MMR IHC deficiency and MSI-H are present in 28\% and $31 \%$ of endometrial malignancies, respectively (13). However, LS gynaecological cancers have MMR expression patterns and MSI similarities with sporadic gynaecological malignancies, making molecular criteria insufficient in triaging potential LS cancers (14). Additionally, more than $50 \%$ of gynecologic cancers with suspected LS based on these molecular criteria lack pathogenic LS-related germline mutations on genetic testing, indicating a high rate of false-positive LS-related MMR deficiency (15).

Clinical and pathologic studies have identified clinical and pathologic characteristics that may be potentially LSdefining. Some of these characteristics include the presence of synchronous or metachronous malignancies, lower BMI, and tumour characteristics such as lower uterine segment (LUS) involvement, endometrioid differentiation, and tumour-infiltrating lymphocytes $(9,16,17)$. Although the inclusion of these clinicopathologic characteristics in the screening workflow may potentially enhance the sensitivity of LS triaging, the proportion of LS gynaecological cancers that demonstrate these clinicopathologic and molecular features have not been ascertained. Their effectiveness in identifying underlying LS in gynecologic cancer is therefore unclear. Thus, this systematic review describes the clinicopathologic features and frequency of LS associated gynecologic cancer. We present the following article in accordance with the PRISMA reporting checklist (available at https://dx.doi.org/10.21037/tcr-21-677).

\section{Methods}

\section{Literature search strategies}

A systematic electronic literature search was performed in the Medline, CINAHL, EMBASE, Google Scholar, and Cochrane Library. The search was based on the PICO strategy of PRISMA: Population (gynaecological cancer), Intervention (Clinical characteristics, histopathology, immunohistochemistry (MLH1, MSH2, MSH6, PMS2) with MLH1 methylation testing, Micro-satellite instability (MSI) analysis, Outcome (screening method positivity) and Comparison (germline mutational analysis). The search criteria included the terms 'gynaecological cancer', 'endometrial cancer', 'cervical cancer', 'lynch syndrome', 'screening', and 'diagnoses. The date of publication was not limited (Figure 1). The protocol of this systematic review was not registered in PROSPERO as it is not mandatory (18).

\section{Inclusion criteria}

Studies were included based on whether they reported the clinico-histopathologic characteristics of confirmed LS gynecologic cancers including tumour morphology and histological features, findings of LS screening, and diagnosis including MMR IHC and/or MSI, MLH1 methylation and genetic testing, and whether they were primary research studies. Studies that reported only clinicopathologic characteristics or molecular characteristics were included if germline mutation testing findings were reported. Studies were excluded if MLH1 methylation and germline mutation 


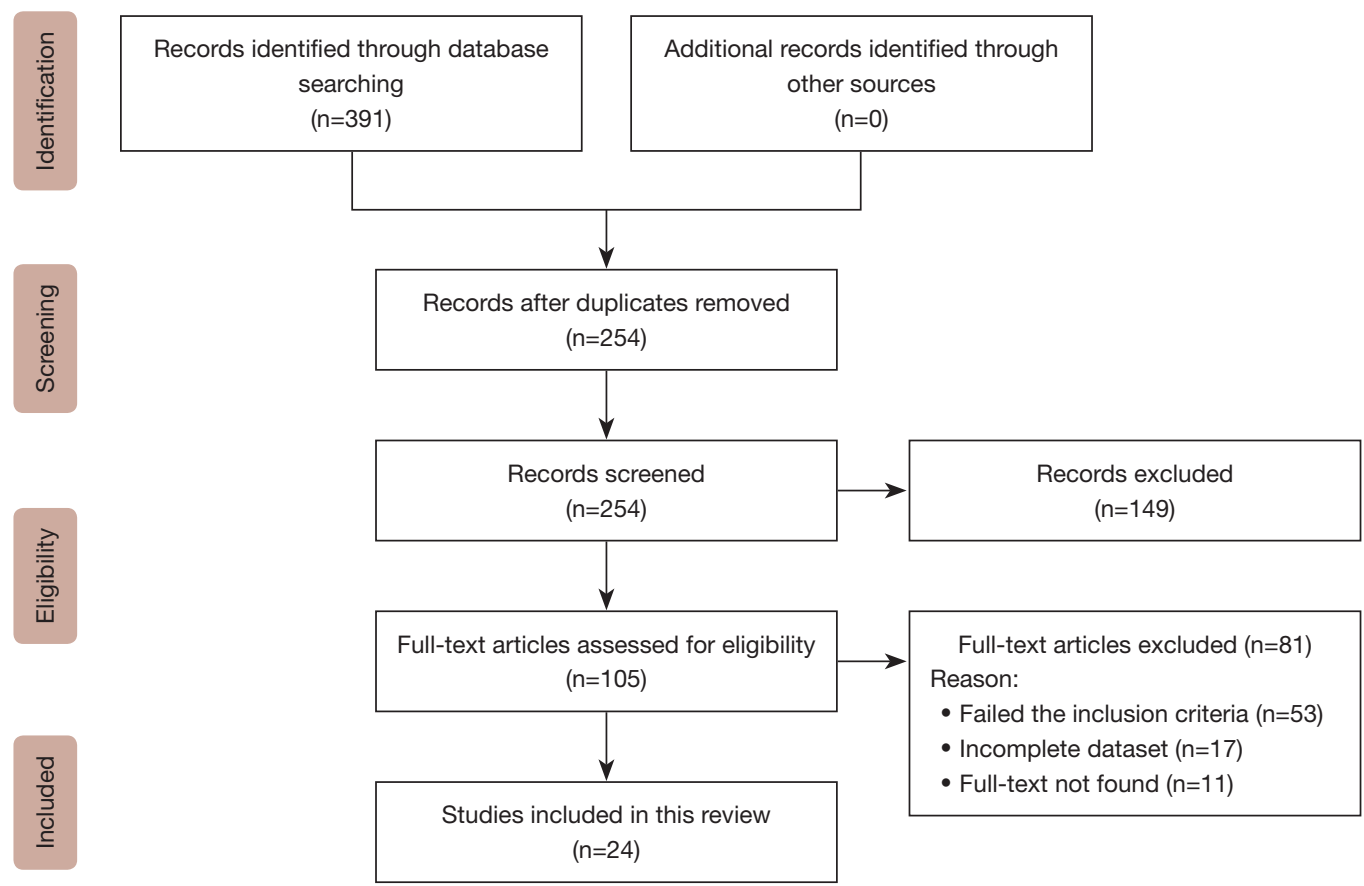

Figure 1 PRISMA flow diagram.

testing were not described.

\section{Data extraction}

Data were extracted from the included studies on the following variables: author, publication year, study design, number of patients with gynecologic (endometrial) cancers screened for LS, the average age at cancer diagnosis, number of patients with methylated and nonmethylated MMR IHC deficient tumours, MSI-MMR IHC concordance rate, number of patients with positive germline mutation testing, BMI, family history of a LS cancer in a first-degree relative (FDR), number of patients with synchronous/metachronous LS cancers, tumour location, FIGO stage, grade, and histological types and features.

\section{Statistical analysis}

The extracted data were analyzed using descriptive statistics, including frequencies, percentages, means, median and standard deviations.

\section{Results}

Total 24 studies, including 14 prospective cohort and
7 retrospective cohort studies, 1 case report, one casecontrol and one prospective-retrospective study, were reviewed. Nineteen studies described both clinicopathologic and molecular characteristics of LS gynaecological cancers. Four and only one studies focused alone on clinicopathologic features and molecular characteristics, respectively.

\section{Clinicopatbologic characteristics}

A total of 22 studies described the clinicopathologic characteristics of LS gynecologic cancers. The average age at diagnosis was $49.94 \pm 4.34$ years with a range of 20-79 years. Six studies reported Lynch-syndrome gynecologic cancer in women aged 30 years and below. The body mass index (BMI) was reported in seven studies. The average BMI was $26.07 \pm 3.77 \mathrm{~kg} / \mathrm{m}^{2}$ with a range of $20.3-$ $32.2 \mathrm{~kg} / \mathrm{m}^{2}$. A family history of LS in endometrial cancer was reported in 12 studies. Synchronous or metachronous LS cancers were reported in 12 studies (Table 1).

Tumour location in endometrial cancers was described in eight studies. Out of 161 cases, the tumour was in the LUS in $28(17.39 \%)$ cases in these studies. A variable number of studies reported tumour histology. Out of 341 cases, maximum studies (70.79\%) were of endometrioid type. Mixed 
Table 1 Clinical characteristics

\begin{tabular}{lc}
\hline Variable ( $\mathrm{n}=$ population) & Frequency $\mathrm{n}(\%) / \mathrm{S} . \mathrm{D}$ \\
\hline Lynch syndrome gynecologic cancers & $517(100.00)$ \\
Median age & $\begin{array}{c}49.94 \pm 4.34 \text { years } \\
\text { (range: } 20-79 \text { years) }\end{array}$ \\
Median BMI ( $\mathrm{n}=7)$ & $26.07 \pm 3.77 \mathrm{~kg} / \mathrm{m}^{2}$ \\
& (range: $\left.20.3-32.2 \mathrm{~kg} / \mathrm{m}^{2}\right)$ \\
History of Lynch syndrome cancer in an & $84(75.68)$ \\
FDR ( $\mathrm{n}=111)$ & \\
Synchronous cancer ( $\mathrm{n}=335)$ & $68(20.30)$ \\
\hline
\end{tabular}

BMI, body mass index; FDR, first degree relative. Percentages are based on sample sizes pooled from studies in which the specific clinical characteristic and its frequency was described. BMI was described in 7 studies.

Table 2 Morphologic and histopathologic characteristics

\begin{tabular}{|c|c|}
\hline Tumor characteristic (population) & Frequency, n (\%) \\
\hline Lower uterine segment location $(n=161)$ & 28 (17.39) cases \\
\hline Average age & $53.1 \pm 7.4$ years \\
\hline \multicolumn{2}{|l|}{ Histology } \\
\hline Endometrioid $(n=341)$ & $242(70.97)$ \\
\hline Serous $(n=204)$ & $17(8.33)$ \\
\hline Clear cell $(n=256)$ & $35(13.67)$ \\
\hline Mixed $(n=284)$ & $30(10.56)$ \\
\hline Other $(n=232)$ & $21(9.05)$ \\
\hline Total endometrial cancer & 345 \\
\hline \multicolumn{2}{|l|}{ FIGO stage } \\
\hline Stage I $(n=243)$ & $173(71.19)$ \\
\hline Stage II $(n=179)$ & $27(15.08)$ \\
\hline Stage III/IV (n=190) & $37(19.47)$ \\
\hline \multicolumn{2}{|l|}{ Grade } \\
\hline I $(n=184)$ & $87(47.28)$ \\
\hline II $(n=166)$ & $43(25.90)$ \\
\hline III $(n=131)$ & $38(29.01)$ \\
\hline \multicolumn{2}{|l|}{ Histological characteristics } \\
\hline Tumor infiltrating lymphocytes ( $\mathrm{n}=78)$ & $20(25.64)$ \\
\hline Peritumoral lymphocytes $(n=61)$ & $27(44.26)$ \\
\hline Lympho-vascular invasion $(\mathrm{n}=23)$ & $13(56.52)$ \\
\hline
\end{tabular}

Percentages are based on sample sizes pooled from studies in which the frequency of the specific morphologic and histopathologic characteristic was described. histology tumours consisted mainly of endometrioid, serous, or clear cell features. They consisted of other histological types, including carcinosarcoma, mucinous, adenosquamous, neuroendocrine, yolk sac, and Mullerian tumour in order of frequency. Among 612 cases, the majority (71.19\%) had stage I disease, and among 481 cases, the majority (47.28\%) had grade 1 tumours. Out of 78 patients, 20 (25.64\%) had tumours with tumour-infiltrating lymphocytes. Twentyseven $(44 \%)$ of 61 patients had tumours with peritumoral lymphocytes. The lymphovascular invasion was reported in $13(56.52 \%)$ of 23 cases (Table 2).

\section{Molecular characteristics}

Twenty studies had complete data on MMR IHC and germline mutation testing findings. A total of 4,247 cases of gynecologic cancer were screened through IHC, MSI, and MLH1 promoter methylation testing. Six hundred twentyeight cases of IHC deficiency without MLH1 methylation. MLH1 methylation findings were reported in 628 patients among 15 studies.

Out of 724 cases with MLH1 deficiency, 628 (86.74\%) cases had MLH1 promoter methylation while 96 (13.26\%) were non-methylated. Among the 649 cases with MMR IHC deficiency, unmethylated MLH1 deficiency was present in $96(14.79 \%)$ cases. PMS2 expression was tested in 17 studies demonstrating 114 cases. MMR-MSI concordance rate findings were available in 15 studies. The average concordance rate was $30.37 \%$ (Table 3).

Among the 649 MMR IHC deficient cases, 336 (51.77\%) cases underwent genetic testing, $162(48.2 \%)$ of the tested cases were confirmed to have pathogenic LS germline mutations. The germline mutations in cases with confirmed LS were reported in 20 studies (160 cases). Of 160 cases with confirmed germline mutations, MLH1 was mutated in $31(19.5 \%)$ cases, while PMS2 was mutated in $14(8.8 \%)$ cases. MSH2 and MSH6 were mutated in 64 (40.2\%) and $51(32.1 \%)$ cases, respectively (Table 4).

\section{Discussion}

A total of 24 studies reporting the clinicopathologic and molecular features of 236 genetically confirmed LS gynecologic cancers. Because of the high proportion of endometrial cancers in the reviewed studies, i.e., $4,210(99.12 \%)$ (Table 3), 'endometrial cancer' is used synonymously with 'gynecologic cancer' in some sections of this review. 
Table 3 MMR IHC

\begin{tabular}{lc}
\hline Population $(\mathrm{n})$ & Number $(\%)$ \\
\hline Screened Lynch syndrome cancers & 4,247 \\
Endometrial cancers & $4210(99.12)$ \\
Synchronous cancers & $22(0.88)$ \\
MLH1 deficient ( $\mathrm{n}=4,247)$ & $724(17.04)$ \\
MLH 1 deficient methylated & $628(86.74)$ \\
MLH1 deficient non-methylated & $96(13.26)$ \\
Non-methylated MMR IHC Deficient $(\mathrm{n}=4,247)$ & $649(15.28)$ \\
Non-methylated MMR IHC Deficient & \\
MLH1 deficient & $96(14.79)$ \\
PMS2 deficient & $114(17.56)$ \\
MSH2 deficient & $173(26.65)$ \\
MSH6 deficient & $266(40.98)$ \\
MMR IHC vs. MSI Concordance rate & $30.37 \%$ \\
Endometrial cancer with Lynch Syndrome (n=345) & $236(68.40)$ \\
\hline
\end{tabular}

MMR IHC, mismatch repair protein immunohistochemistry; MSI, Microsatellite instability. Percentages are based on sample sizes pooled from studies in which the specific molecular screening finding was described. PMS2 was tested in 114 of the 649 MMR IHC deficient tumors.

Table 4 Germline mutation characteristics

\begin{tabular}{lc}
\hline Population $(n)$ & Frequency $n(\%) / S . D$ \\
\hline $\begin{array}{l}\text { MMR IHC Deficient cases that underwent } \\
\text { genetic testing }(\mathrm{n}=649)\end{array}$ & $336(51.77)$ \\
$\begin{array}{l}\text { Tested MMR IHC Deficient cases with } \\
\text { confirmed Lynch syndrome }(\mathrm{n}=336)\end{array}$ & $162(48.2)$ \\
Germline mutation status ( $\mathrm{n}=159)$ & \\
MLH1 Mutation & $31(19.5)$ \\
PMS2 Mutation & $14(8.8)$ \\
MSH2 & $64(40.2)$ \\
MSH6 & $51(32.1)$ \\
\hline
\end{tabular}

MMR ICH, mismatch repair protein immunohistochemistry. Only 336 out of 469 MMR IHC deficient tumors underwent germline mutation testing. Specific germline mutations were described in 159 of 162 cancers with confirmed Lynch syndrome. One tumor had $\mathrm{MSH} 2$ and $\mathrm{MSH} 6$ germline mutations.

The average age at diagnosis of LS gynecologic cancer was $49.94 \pm 4.34$ years (Table 1). Young age at diagnosis of gynecologic cancer is an established characteristic of LS based on the findings of previous studies. Mas-Moya et al. found that patients who underwent genetic testing were younger (51 years) compared to the patients who did not undergo genetic testing (59 years) following molecular screening (19). In this review, the average age at diagnosing gynaecological cancers in patients who underwent molecular screening was $53.1 \pm 7.4$ years. This review indicates the need for further risk stratification based on diagnosis among at-risk women in the $5^{\text {th }}$ and $6^{\text {th }}$ decades of life.

The average BMI of the patients with confirmed LS was $26.07 \pm 3.77 \mathrm{~kg} / \mathrm{m}^{2}$ (Table 1). Previous studies have found an equivocal association between BMI and LS gynecologic cancers (20). However, the lower BMI obtained in this review compared to previous studies suggests that a lower BMI may also contribute to stratifying the risk of LS in gynecologic cancer patients. Gordhandas et al. found a statistically significant difference in BMI among patients with mutation-confirmed LS $\left(27.6 \mathrm{~kg} / \mathrm{m}^{2}\right)$ compared to patients with MMR deficiency $\left(32.6 \mathrm{~kg} / \mathrm{m}^{2}\right)$ (21). Similarly, Cohn et al. found that MMR deficient patients who have MSI tumours have a lower BMI $\left(30.5 \mathrm{~kg} / \mathrm{m}^{2}\right)$ than MMR deficient patients with MSS tumours $\left(33.8 \mathrm{~kg} / \mathrm{m}^{2}\right)(22)$, suggesting the possibility of a BMI related risk continuum. Since obesity is an established risk factor for endometrial cancer (23), BMI can be used to assign the risk for LS in gynecologic cancers, particularly endometrial carcinoma.

In an FDR, a family history of LS cancer was found in $84(75.68 \%$ ) (Table 1). However, this proportion may be lower since three of the studies that reported the highest percentage of cases with a history of LS cancer in an FDR included participants from high-risk families. A smaller proportion was obtained in a recent meta-analysis which found that family history-based triaging identifies only $56 \%$ of endometrial cancers with LS (13). Kahn et al. found that family history-dependent screening of endometrial cancers can miss approximately $43 \%$ of LS endometrial cancers (13). This is because most LS gynecologic cancer patients with unknown germline mutation status are likely to be probands.

Sixty-eight of 335 patients had synchronous or metachronous LS malignancies during LS gynecologic cancer diagnosis (Table 1). This proportion may be lower since some of the studies did not distinguish between synchronous and metachronous cancers. Other studies have also demonstrated a similar association. In the study conducted by Lu et al., out of nine patients with synchronous ovarian cancer who underwent genetic testing, only $1(11.1 \%)$ had a germline mutation (24). However, the molecular characteristics of these tumours were not 
reported. Notably, the finding of MMR IHC deficiency in synchronous tumours predicts the existence of underlying LS. All three patients with synchronous tumours and MMR IHC deficiency who underwent germline testing had a pathogenic LS germline mutation (25). Synchronous cancer presentation may therefore complement molecular criteria in defining the risk profile of gynecologic cancer patients for genetic testing.

An LUS location of the LS endometrial cancer was found in $28(17.39 \%)$ of 161 patients (Table 2). In the Westin et al. study, 5 out of 6 MMR IHC deficient women with LUS endometrial cancers who underwent genetic testing were confirmed to have LS (17). As such, the finding of LSassociated molecular characteristics in an LUS endometrial tumour may enhance the identification of LS.

In the reviewed studies, endometrioid differentiation was the most frequent $(70.97 \%)$ histological type (Table 2). Gordhandas et al. found that LS gynecologic cancers typically demonstrate heterogenous histologies (21). However, their study did not cater for the variations in the sample sizes of the studies reporting these characteristics. Since $70-80 \%$ of all endometrial cancers are endometrioid (23), this review supports the need for universal screening for LS in endometrial cancers.

This review finds significant heterogeneity in the histological grades of LS gynecologic malignancies at diagnosis. Regarding tumour stage, the frequency of stage I disease in LS gynecologic cancers at the time of diagnosis was $71.19 \%$ (Table 2). This indicates that most gynecologic cancers caused by underlying LS are not advanced at the time of presentation. The high frequency of stage I cases may be related to the non-indolent clinical pattern of endometrial cancers since most women develop symptoms early during disease presentation (26). Tumour grade is therefore potentially non-efficacious in identifying underlying LS.

Twenty (25.64\%) out of 78 patients had tumours with tumour-infiltrating lymphocytes feature. Peritumoral lymphocytes and lymphovascular invasion occurred in $44.26 \%$ and $56.52 \%$ of the cases. However, these characteristics were reported in small sample sizes (20, 27 and 13 cases, respectively). As such, although gynecologic cancer patients can be selected for genetic testing based on these histologic features, the absence of these features does not exclude underlying LS.

In patients with gynaecological cancers who underwent molecular screening, 649 (15.28\%) cases had MMR IHC deficiency without MLH1 methylation (Table 3). As such, based on the current molecular criteria for triaging LS gynecologic cancers (10), only $15.28 \%$ of all gynecologic cancers are likely to be selected for germline mutation testing. Among 4,247 patients, 724 (17.04\%) had a loss of MLH1. The frequency of methylation in tumours with loss of expression of MLH1 was $86.74 \%$ (628 patients). Kahn et al. obtained similar findings with a frequency of MLH1 loss of $20 \%$ and an MLH1 methylation rate of $86 \%$ (13). MLH1 methylation appears to have a significant correlation with an MSI-H status. Ten out of 11 MLH1 methylated cases in the Lu et al. study were MSI-H and germline mutation-negative (24). In two studies, an MLH1 methylation rate of $100 \%$ was found $(27,28)$. Since one of the major hindrances of universal molecular screening is the prohibitive cost of MSI analysis (10), MLH1 methylation testing in tumours with isolated loss of expression of MLH1 may improve the feasibility of screening, precluding the need for MSI analysis.

According to this review, although the loss of expression of MLH1 in gynecologic cancers mainly results from promoter hypermethylation, the gene is still involved in a proportion of unmethylated MMR IHC deficient tumours comparable to both PMS2 and MSH2. Among unmethylated MMR IHC secondary tumours, loss of expression of MSH6 was the most frequent (40.98\%) abnormality. However, this review confirms the findings of previous studies that have shown a predominant implication of the MSH2 and MSH6 genes in abnormal IHC MMR (29).

Approximately $48.2 \%$ of the MMR IHC deficient tumours that underwent germline testing confirmed LS. Mills et al. obtained a similar concordance rate $(46.7 \%)$ between the two diagnostic workups. Several germline mutation variants of undetermined significance have been identified in MMR deficient tumours, which supports the possibility of an unelucidated Lynch-like syndrome $(3,28)$.

MSH2 was the most frequently mutated gene (40\%), followed by MSH6 (32\%). This indicates that the majority of LS cases arise from mutations within the MSH2/6 heterodimer. MLH1 and PMS2 were mutated in $19.5 \%$ and $8.8 \%$ of the cases. The frequency of each germline mutation obtained in this study mirrors the findings of other metaanalytic studies (13).

Our finding of endometrial cancer with LS rate was $60.64 \%$, according to the observation of $40-60 \%$ in Meyer et al. study (30). This is the first study to examine the frequency of LS-associated clinicopathologic and molecular characteristics in gynecologic cancers with mutationconfirmed LS. Previous studies have predominantly 
described these characteristics in MMR deficient and sporadic cancers $(13,21)$. This study also used the individual sample sizes of the included studies to derive the proportions of LS gynecologic cancers exhibiting the clinicopathologic features of interest, which increased the accuracy of the findings. Additionally, this review included studies in which methylation findings were reported, hence the methylation status of all patients, and consequently, their valid eligibility for genetic testing was known.

\section{Limitations}

One limitation of this review is the small sample of non-endometrial gynecologic cancers. This resulted from the shortage of studies with complete data on the clinicopathologic and molecular characteristics of endometrial gynecologic cancers. Similarly, the suboptimal rate of genetic testing among eligible patients yielded a small number of patients with a positive germline mutation. Reasons for low uptake of gene testing included a loss of follow-up, patient anxiety, rejection of testing, and failure of physicians to refer patients $(27,31)$. However, since this review used a large sample size (4,247 patients), and the overall uptake rate of genetic testing was $73.67 \%$, the small number of confirmed LS cases indicates the low prevalence of LS in gynecologic cancers.

Similarly, in two studies, some of the participants were referred for genetic counselling based on clinical criteria, which may affect the accuracy of the concordance rate between tumour testing and genetic testing $(14,17)$. However, in one of the two studies, all the referred patients were later found to have LS-associated molecular features (17). In contrast, in the other study, most of the referrals met the molecular criteria (14).

\section{Conclusions}

This systematic review found that young age at diagnosis, normal or high normal BMI, family history of Lynch cancer syndrome, stage I disease, endometrioid differentiation, low-grade lesion, peritumoral lymphocytes, and lymphovascular invasion are frequent clinicopathologic characteristics of LS gynaecological cancers. The frequency of synchronous malignancies, LUS tumour location, and tumour-infiltrating lymphocytes are low to intermediate. Similarly, the high rate of involvement of the MSH2/MSH6 heterodimer in both MMR IHC deficiency and LS germline mutations, alongside the high frequency of non-LS-related
MLH1 loss, indicates that MSH2/MSH6 abnormality is a high-risk molecular characteristic.

\section{Acknowledgments}

Funding: This project was supported by the National Natural Science Foundation of China (81671409), Beijing Natural Science Foundation of China (7202239), and Beijing Municipal Administration of Hospitals Incubating Program (PX2017040).

\section{Footnote}

Reporting Checklist: The authors have completed the PRISMA reporting checklist. Available at https://dx.doi. org/10.21037/tcr-21-677

Conflicts of Interest: All authors have completed the ICMJE uniform disclosure form (available at https://dx.doi. org/10.21037/tcr-21-677). All authors report this project was supported by the National Natural Science Foundation of China (81671409), Beijing Natural Science Foundation of China (7202239), and Beijing Municipal Administration of Hospitals Incubating Program (PX2017040). The authors have no other conflicts of interest to declare.

Ethical Statement: The authors are accountable for all aspects of the work in ensuring that questions related to the accuracy or integrity of any part of the work are appropriately investigated and resolved.

Open Access Statement: This is an Open Access article distributed in accordance with the Creative Commons Attribution-NonCommercial-NoDerivs 4.0 International License (CC BY-NC-ND 4.0), which permits the noncommercial replication and distribution of the article with the strict proviso that no changes or edits are made and the original work is properly cited (including links to both the formal publication through the relevant DOI and the license). See: https://creativecommons.org/licenses/by-nc-nd/4.0/.

\section{References}

1. Bonadona V, Bonaïti B, Olschwang $\mathrm{S}$, et al. Cancer risks associated with germline mutations in MLH1, MSH2, and MSH6 genes in Lynch syndrome. JAMA 2011;305:2304-10.

2. Lu KH, Dinh M, Kohlmann W, et al. Gynecologic cancer as a "sentinel cancer" for women with hereditary 
nonpolyposis colorectal cancer syndrome. Obstet Gynecol 2005;105:569-74.

3. Mills AM, Longacre TA. Lynch Syndrome: Female Genital Tract Cancer Diagnosis and Screening. Surg Pathol Clin 2016;9:201-14.

4. Antill YC, Dowty JG, Win AK, et al. Lynch syndrome and cervical cancer. Int J Cancer 2015;137:2757-61.

5. Niessen RC, Hofstra RM, Westers H, et al. Germline hypermethylation of MLH1 and EPCAM deletions are a frequent cause of Lynch syndrome. Genes Chromosomes Cancer 2009;48:737-44.

6. Rumilla K, Schowalter KV, Lindor NM, et al. Frequency of deletions of EPCAM (TACSTD1) in MSH2-associated Lynch syndrome cases. J Mol Diagn 2011;13:93-9.

7. Bartley AN, Luthra R, Saraiya DS, et al. Identification of cancer patients with Lynch syndrome: clinically significant discordances and problems in tissue-based mismatch repair testing. Cancer Prev Res (Phila) 2012;5:320-7.

8. Frolova AI, Babb SA, Zantow E, et al. Impact of an immunohistochemistry-based universal screening protocol for Lynch syndrome in endometrial cancer on genetic counseling and testing. Gynecol Oncol 2015;137:7-13.

9. Kwon JS, Scott JL, Gilks CB, et al. Testing women with endometrial cancer to detect Lynch syndrome. J Clin Oncol 2011;29:2247-52.

10. Djordjevic B, Broaddus RR. Laboratory Assays in Evaluation of Lynch Syndrome in Patients with Endometrial Carcinoma. Surg Pathol Clin 2016;9:289-99.

11. Sahnane N, Magnoli F, Bernasconi B, et al. Aberrant DNA methylation profiles of inherited and sporadic colorectal cancer. Clin Epigenetics 2015;7:131.

12. Hampel H, Frankel W, Panescu J, et al. Screening for Lynch syndrome (hereditary nonpolyposis colorectal cancer) among endometrial cancer patients. Cancer Res 2006;66:7810-7.

13. Kahn RM, Gordhandas S, Maddy BP, et al. Universal endometrial cancer tumor typing: How much has immunohistochemistry, microsatellite instability, and MLH1 methylation improved the diagnosis of Lynch syndrome across the population? Cancer 2019;125:3172-83.

14. Carnevali I, Libera L, Chiaravalli A, et al. Somatic Testing on Gynecological Cancers Improve the Identification of Lynch Syndrome. Int J Gynecol Cancer 2017;27:1543-9.

15. Buchanan DD, Rosty C, Clendenning M, et al. Clinical problems of colorectal cancer and endometrial cancer cases with unknown cause of tumor mismatch repair deficiency (suspected Lynch syndrome). Appl Clin Genet 2014;7:183-93.
16. Soslow RA. Practical issues related to uterine pathology: staging, frozen section, artifacts, and Lynch syndrome. Mod Pathol 2016;29 Suppl 1:S59-77.

17. Westin SN, Lacour RA, Urbauer DL, et al. Carcinoma of the lower uterine segment: a newly described association with Lynch syndrome. J Clin Oncol 2008;26:5965-71.

18. Tawfik GM, Giang HTN, Ghozy S, et al. Protocol registration issues of systematic review and meta-analysis studies: a survey of global researchers. BMC Med Res Methodol 2020;20:213.

19. Mas-Moya J, Dudley B, Brand RE, et al. Clinicopathological comparison of colorectal and endometrial carcinomas in patients with Lynch-like syndrome versus patients with Lynch syndrome. Hum Pathol 2015;46:1616-25.

20. Moline J, Mahdi H, Yang B, et al. Implementation of tumor testing for lynch syndrome in endometrial cancers at a large academic medical center. Gynecol Oncol 2013;130:121-6.

21. Gordhandas S, Kahn RM, Gamble C, et al. Clinicopathologic features of endometrial cancer with mismatch repair deficiency. Ecancermedicalscience 2020;14:1061.

22. Cohn DE, Pavelka JC, Frankel WL, et al. Correlation between patient weight and defects in DNA mismatch repair: is this the link between an increased risk of previous cancer in thinner women with endometrial cancer? Int J Gynecol Cancer 2008;18:136-40.

23. Burbos N, Musonda P, Giarenis I, et al. Predicting the risk of endometrial cancer in postmenopausal women presenting with vaginal bleeding: the Norwich DEFAB risk assessment tool. Br J Cancer 2010;102:1201-6.

24. Lu KH, Schorge JO, Rodabaugh KJ, et al. Prospective determination of prevalence of lynch syndrome in young women with endometrial cancer. J Clin Oncol 2007;25:5158-64.

25. Walsh CS, Blum A, Walts A, et al. Lynch syndrome among gynecologic oncology patients meeting Bethesda guidelines for screening. Gynecol Oncol 2010;116:516-21.

26. Amant F, Moerman P, Neven P, et al. Endometrial cancer. Lancet 2005;366:491-505.

27. Cameron A, Chiarella-Redfern H, Chu P, et al. Universal Testing to Identify Lynch Syndrome Among Women With Newly Diagnosed Endometrial Carcinoma. J Obstet Gynaecol Can 2020;42:137-43.

28. Mills AM, Sloan EA, Thomas M, et al. Clinicopathologic Comparison of Lynch Syndrome-associated and "Lynch-like" Endometrial Carcinomas Identified on 
Universal Screening Using Mismatch Repair Protein Immunohistochemistry. Am J Surg Pathol 2016;40:155-65.

29. Ryan NAJ, Glaire MA, Blake D, et al. The proportion of endometrial cancers associated with Lynch syndrome: a systematic review of the literature and meta-analysis. Genet Med 2019;21:2167-80.

Cite this article as: Yang Z, Liu X, Yang X, Liao QP. Screening and identification of Lynch syndrome: a systematic review of the frequency of Lynch syndrome-associated clinicopathologic and molecular characteristics in Lynch syndrome gynecologic cancers. Transl Cancer Res 2021;10(10):4523-4531. doi: 10.21037/ tcr-21-677
30. Meyer LA, Broaddus RR, Lu KH. Endometrial cancer and Lynch syndrome: clinical and pathologic considerations. Cancer Control 2009;16:14-22.

31. Egoavil C, Alenda C, Castillejo A, et al. Prevalence of Lynch syndrome among patients with newly diagnosed endometrial cancers. PLoS One 2013;8:e79737. 